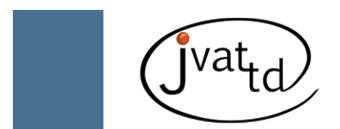

\title{
Diagnosis of paracoccidioidomycosis in patients attended in routine services of a university hospital
}

Moreto TC (1), Mendes RP (2)

(1) Graduate Program, Botucatu Medical School, São Paulo State University (UNESP - Univ Estadual Paulista), Botucatu, São Paulo State, Brazil; (2) Department of Tropical Diseases, Botucatu Medical School, São Paulo State University (UNESP - Univ Estadual Paulista), Botucatu, São Paulo State, Brazil.

Abstract: Identification of appropriate laboratory procedures to confirm a clinical hypothesis is important in a routine service for paracoccidioidomycosis patients and constituted the objective of this study. Medical charts and laboratory records of 401 paracoccidioidomycosis patients attended at the Department of Tropical Diseases in the Botucatu Medical School (São Paulo, Brazil) from 1974 to 2008 were reviewed. Direct mycological examination (DM), cell block preparation (CB), histopathological examination (HP) and specific serum antibody levels evaluated through double agar gel immunodiffusion test (ID) were analyzed before treatment. Statistical analysis: the comparison between proportions for dependent or independent populations was performed by the McNemar's or binomial test. The agreement between methods was evaluated using the kappa coefficient. The relationship of more than two dependent populations was made by the Cochran test. The comparison of multiple proportions was done by Tukey test and, for two proportions, by Z test. In order to study the association between qualitative variables the chi-square test was employed. For quantitative variables, whose objective was to compare groups, the nonparametric Kruskal-Wallis test was used. The Statistical Analysis System software (SAS), version 6.12, was utilized. Significance was set up at $p<0.05$. Males (88.0\%) and chronic form (76.8\%) were predominat. Patient distribution according to the period of assistance presented no differences. Typical $P$. brasiliensis yeast forms were identified in clinical specimens in $86 \%$ of the patients while $14 \%$ of them showed only a positive serological test. Direct mycological examination carried out in 51 different tissue specimens showed $74.5 \%$ sensitivity. The sensitivity was $62.5 \%$ in 112 sputum samples. Cell block preparation carried out in 483 sputum samples showed $55.3 \%$ sensitivity. Histopathological examination performed in 239 tissue samples from different organs revealed $96.7 \%$ sensitivity. Serological tests carried out in 351 patients and 200 healthy controls paired according to gender and showed $90 \%$ sensitivity, $100 \%$ specificity, $100 \%$ positive predictive value, $85 \%$ negative predictive value and $94 \%$ accuracy. Comparisons $2 \times 2$ of laboratory measurements carried out in the same patient showed that sensitivity decreases from HP to serology to $C B$ and $D M$; the last two assays showed no differences in sensitivity. Serum samples from 32 patients with confirmed paracoccidioidomycosis but negative immunodiffusion before treatment were evaluated. As controls, positive sera from 32 additional confirmed patients were analyzed. These assays were carried out at the Research Laboratory of Tropical Diseases, Botucatu Medical School, UNESP, and at the Adolfo Lutz Institute, São Paulo. ID was performed using culture filtrate antigens from $\mathrm{Pb}-113$ prepared at the Laboratory of Clinical Mycology, UNESP, Araraquara (IDr) and from Pb-113 (ID1) and Pb-B-339 (ID2) prepared at the Adolfo Lutz Institute. Immunoblotting was also carried out using Pb-113 (IB1) and PbB-339 (IB2) strains. Our findings showed that ID performed at the Research Laboratory of Tropical Diseases presented no difference in positivity among the three antigens. ID performed at Adolfo Lutz Institute presented no difference in positivity between ID1 and ID2, but results were higher than in IDr. Reproducibility between laboratories was observed with ID1 and ID2, but IDr presented a somewhat higher sensitivity at the Research

T. C. Moreto was advised by Professor Rinaldo Poncio Mendes, and submitted this thesis for her Masters in Tropical Diseases at the Botucatu Medical School, São Paulo State University (UNESP - Univ Estadual Paulista), Botucatu, São Paulo State, Brazil, 2010. 
Laboratory of Tropical Diseases. There was no difference in sensitivity for IB1-gp43, IB2-gp70 and IB2-gp43. However, it was higher than that found for IB2-gp70. ID positivity was lower than IB1-gp43, IB2-gp43 and IB2-gp43 recognition, but higher than IB2-gp70. This study shows that $P$. brasiliensis can be identified in almost all the cases, mainly by histopathological examination, and the importance of the double agar gel immunodiffusion test in a routine service, including for long periods, with different technicians, biologists and pathologists. Our findings also show that negative ID serum should be submitted to immunoblotting using $\mathrm{Pb}-113$ to evaluate the gp70 because of its high sensitivity, specificity, and positive and negative predictive values.

Key words: paracoccidioidomycosis, diagnosis, routine services, immunodiffusion, immunoblotting.

\section{COPYRIGHT}

(c) CEVAP 2010

\section{SUBMISSION STATUS}

Received: February 11, 2010.

Accepted: February 11, 2010.

Abstract published online: February 12, 2010.

Full paper published online: August 31, 2010.

\section{CORRESPONDENCE TO}

TALÍSIA COLLACHITI MORETO, Departamento de Doenças Tropicais e Diagnóstico por Imagem, Faculdade de Medicina de Botucatu, UNESP, Botucatu, SP, 18.618-000, Brasil. Phone: +55 143811 6212. Email: tamoreto@yahoo.com.br. 